\title{
Immediate angiographic residual shunt using the Nit-Occlud device for patent ductus arteriosus closure
}

\author{
Gurumurthy Hiremath, Varun Aggarwal, Katy Soule, John L. Bass \\ Department of Pediatrics, Division of Pediatric Cardiology, University of Minnesota, Masonic Children's Hospital, Minneapolis, United States
}

Adv Interv Cardiol 2020; 16, 4 (62): 460-465

DOI: https://doi.org/10.5114/aic.2020.101772

\begin{abstract}
A bstract
Introduction: The Nit-Occlud PDA device is a newer coil-type device with a high degree of efficacy and safety. There are concerns about the high incidence of immediate angiographic residual shunt with this device.

Aim: To compare immediate angiographic residual shunts and their outcomes following PDA device closure with the Nit-Occlud device.

Material and methods: A single-institution, retrospective chart review of PDA closures was performed. Thirty patients who underwent Nit-Occlud PDA closure were compared with 34 patients who underwent PDA closure with an Amplatzer Duct Occluder-1 (ADO-1) and 25 patients who underwent PDA closure with coils.

Results: The three groups were similar in age, weight, and procedural characteristics. The PDA dimensions were smaller in the coils group. Technical success in the ADO-1 and Nit-Occlud groups was 100\%. A small angiographic residual shunt was seen more often in the Nit-Occlud group (70\%) than in the ADO-1 (59\%) and coils (26\%) groups ( $p=0.005)$. Most residual shunts in the Nit-Occlud group disappeared in the echocardiogram performed $4 \mathrm{~h}$ later (90\% echocardiographic closure). Echocardiographic closure (100\%) was seen at 2 months and 6 months in the Nit-Occlud group. No correlation was noted between the angiographic residual shunt and Nit-Occlud device orientation with respect to the ductus, the device-ductal angle or the number of loops at the pulmonary artery end.

Conclusions: Despite the higher immediate angiographic residual shunt rate in the Nit-Occlud group than the other groups, high echocardiographic closure rates were seen within hours after device closure, which persisted at follow-up. The angiographic residual shunt is not related to the device orientation and should not be a deterrent in using this device.
\end{abstract}

Key words: patent ductus arteriosus, Nit-Occlud device, residual shunt.

Sum mary

The immediate angiographic residual shunt is more common with the Nit-Occlud device compared to ADO-1 and other coils and is not related to device position or orientation with respect to the PDA. Angiographic residual shunts do not correlate with echocardiographic residual shunts and should not be a deterrent to using this device

\section{Introduction}

Persistent patent ductus arteriosus (PDA) is a common defect and occurs in $5-10 \%$ of all congenital heart defects [1]. Persistent PDA is closed either by surgery or by a percutaneous transcatheter approach to eliminate the left-to-right shunt, prevent pulmonary vascular disease or eliminate the risk of endocarditis [2]. Transcatheter closure was first described by Portsmann et al. in 1967 and is the preferred method of closure of the PDA [3]. Historically, a variety of transcatheter devices have been used to close a PDA, starting with the Ivalon plug, the umbrella-type PDA occluder, a variety of coils, vascular plugs and the Amplatzer PDA device.

There are currently four transcatheter devices that have gained Food and Drug Administration (FDA) approval for PDA closure in the United States. These include the Amplatzer Duct Occluders (ADO) type 1 and 2, the Nit-Occlud PDA device and the most recent addition, the Amplatzer Piccolo occluder (specifically indicated for premature infants). In addition to these devices, a variety of coils and Amplatzer vascular plugs are routinely used "off label" to close PDAs. The Nit-Occlud PDA device (PFM

\section{Corresponding author:}

Gurumurthy Hiremath MD, Department of Pediatrics, Division of Pediatric Cardiology, University of Minnesota, Masonic Children's Hospital, Minneapolis, MN 55446, United States, phone: 6126262467, e-mail: hiremath@umn.edu

Received: 10.06.2020, accepted: 8.10.2020. 
Medical, Cologne, Germany) is a coil-type device with a controlled release mechanism that is designed to close small to moderate ductus primarily through a venous approach. The results of the multicenter Nit-Occlud PDA pivotal study and the continuing access study showed 95\% technical success at implantation and $96.8 \%$ complete echocardiographic closure rates at 12 months, with a very low rate of adverse events [4, 5].

It is standard practice during transcatheter PDA closure to perform an angiogram in the aorta to look at device position with respect to PDA and to assess the immediate angiographic residual shunt. The immediate angiographic residual shunt is more frequent with the Nit-Occlud device compared to Gianturco coils, Flipper coils, and ADO-1 [6]. Echocardiographic residual shunts at 6 months and 12 months are, however, infrequent. Nevertheless, any immediate angiographic residual shunt is unsatisfactory to an interventional cardiologist. There are limited data on the angiographic residual shunt rates with the Nit-Occlud device in the pediatric population.

\section{Aim}

We decided to look at our institutional experience with the Nit-Occlud device with a special focus on the immediate angiographic residual shunt and its implications.

\section{Material and methods}

This is a retrospective review of PDA device closures at a single institution from 2011 to 2015 performed after approval from the Institutional Review Board. PDA closures that were performed using the Nit-Occlud device and the ADO-1 device and coils were included. The coils group included a combination of Gianturco coils, Flipper coils, and MReye Embolization Coils (Cook Medical, Bloomington, IN). Amplatzer vascular plugs and ADO-2 were not used frequently to close PDA at our institution and hence were excluded from the study.
Available catheterization reports, echocardiograms, and angiograms were reviewed. A review of the electronically stored catheterization reports was performed to obtain measurements of the PDA (minimal diameter, maximal diameter at the aortic end, and length). The principal investigator reviewed all angiograms to reconfirm the Krichenko classification [7] of the ductus and the immediate angiographic residual shunt. The immediate angiographic residual shunt was classified as follows: tiny, if a small puff of contrast was seen limited to the area of the device; small, if it opacified the main pulmonary artery without outlining the valve; and moderate, if it outlined the pulmonary valve $[8,9]$. The angle between the long axis of the ductus and the device (device-ductal angle) was measured as seen in Figure 1 based on the appearance on a single lateral plane angiogram after the release of the device. The number of loops of the device that extended into the pulmonary artery was also noted. Complications were documented from the catheterization database. The electronic health records and echocardiographic database were reviewed for follow-up data on the patients. The length of follow-up, presence of residual shunt at discharge echo, and presence of any residual shunt at late follow-up were noted. The echocardiographic residual shunt was determined by color flow Doppler in the parasternal short axis and suprasternal views.

\section{Statistical analysis}

Statistical analysis was performed using IBM SPSS statistical software version 23. Baseline data were described using nonparametric descriptors. The three groups (Nit-Occlud, 'Coils' and ADO-1) were compared for demographic, procedural, outcome characteristics and residual shunts using Kruskal-Wallis and $\chi^{2}$ tests as appropriate. Further evaluation was performed to compare the orientation and positioning of the Nit-Occlud
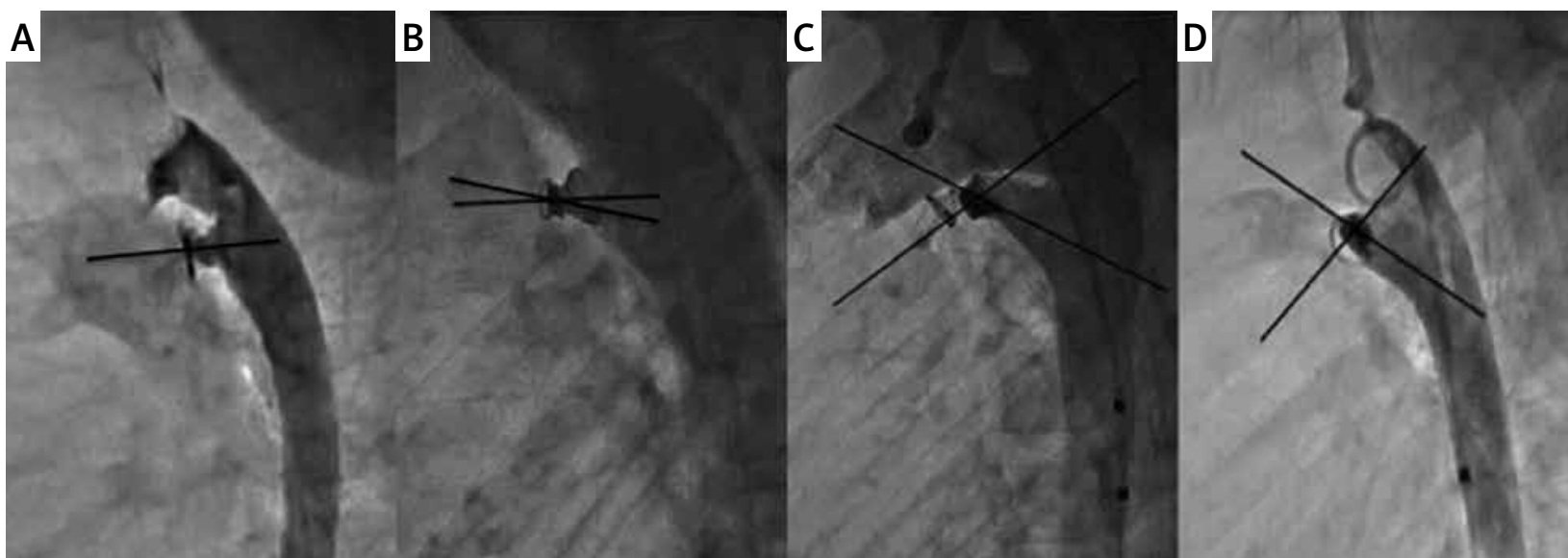

Figure 1. Orientation of the Nit-Occlud device with the length of the ductus. Legend: Varying orientations of Nit-Occlud device with the ductus. Varying device-ductal angles ( $0^{\circ}$ in $\mathbf{A}, 12.5^{\circ}$ in $\mathbf{B}, 39^{\circ}$ in $\mathbf{C}$ and $88^{\circ}$ in $\left.\mathbf{D}\right)$ and a varying number of loops in the pulmonary artery 
device with the incidence of immediate residual shunt on angiogram and at follow-up.

\section{Procedural technique}

Baseline aortic angiography was performed in all cases to assess ductal morphology and dimensions using a pigtail catheter in the proximal descending aorta in biplane projections (right anterior oblique and caudal projection on the frontal plane and straight lateral projection on the lateral plane). Measurements performed on the PDA included minimal diameter, maximal diameter, diameter at the aortic ampulla, and length. The type of device and the size were based on the shape and size of the PDA, attending preference, and published 'instructions for use'. For Nit-Occlud, the device was chosen so that the largest diameter of the coil was at least 3-4 mm larger than the minimal PDA diameter and no more than $2 \mathrm{~mm}$ larger than the aortic ampulla. For ADO-1, the device was selected so that the smaller end of the device measured at least $2 \mathrm{~mm}$ larger than the minimal PDA diameter, while also ensuring that the aortic disk was not too large for the descending aorta next to the PDA. The 0.038-inch diameter embolization coils (Gianturco, Flipper, and MReye) were chosen so that the diameter of the coil loops was at least twice the minimal PDA diameter and the length was adequate to form three-quarters of a loop in the pulmonary artery and 3-4 loops in the aorta as previously described [10]. All Nit-Occlud PDA and ADO-1 devices were deployed via the venous approach. All Gi- anturco, Flipper, and MReye coils were delivered via the retrograde arterial approach. Arterial access was used in all cases for angiography to aid device placement. Angiograms were performed after device placement (before release) and after release of the device to assess device position and residual shunt. Heparin was administered in all patients to maintain an activated clotting time above 220 ms. Per institutional practice, an echocardiogram was performed before the discharge of the patient $4 \mathrm{~h}$ after the procedure, and the same was repeated at follow-up at 2 and 6 months after the procedure.

\section{Results}

Thirty patients underwent PDA device closure with the Nit-Occlud device, 34 with ADO-1 and 25 with Coils during this timeframe. Table I illustrates the demographic and procedural characteristics of the three groups. There were no significant differences in the age and weight of subjects in the three groups. The median procedure time (as assessed by sheath-in to sheath-out time), fluoroscopy time and radiation dose were similar in all 3 groups. The procedure was technically successful in $100 \%$ of the Nit-Occlud cases and ADO- 1 cases. There were 2 immediate failures in the Coils group due to coil embolization. The overall incidence of adverse events was $5.5 \%$. The only complication in the Nit-Occlud group was an episode of bradycardia during anesthesia induction treated with brief chest compressions. The patient had successful PDA closure and was discharged without issues.

Table I. Demographic, procedural and patent ductus arteriosus characteristics of the 3 subgroups of PDA device closure

\begin{tabular}{|c|c|c|c|c|}
\hline Parameter & $\begin{array}{l}\text { Nit-Occlud } \\
(n=30)\end{array}$ & $\begin{array}{c}\text { Coils } \\
(n=25)\end{array}$ & $\begin{array}{l}\text { ADO-1 } \\
(n=34)\end{array}$ & $P$-value \\
\hline Age, median (range) [months] & $47(11-424)$ & $47.3(4.7-246.7)$ & $31.3(7.3-318)$ & $0.54^{\star}$ \\
\hline Weight, median (range) [kg] & $16.2(8.3-78.7)$ & $15.9(3.9-104.4)$ & $14.1(6.6-67.8)$ & $0.58^{*}$ \\
\hline Procedure time, median (range) [min] & $60.5(43-103)$ & $71(34-173)$ & $68.5(40-102)$ & $0.18^{\star}$ \\
\hline Dose area product, median (range) $\left[\mu \mathrm{Gym}^{2}\right]$ & $603(181-12962)$ & $540(139-14941)$ & $758.8(236.5-19687.5)$ & $0.25^{\star}$ \\
\hline Patent ductus arteriosus type: & & & & 0.322 \\
\hline A & $36.7 \%$ & $12 \%$ & $38.2 \%$ & \\
\hline B & $0 \%$ & $0 \%$ & $5.8 \%$ & \\
\hline C & $3.3 \%$ & $4 \%$ & $17.6 \%$ & \\
\hline $\mathrm{D}$ & $0 \%$ & $8 \%$ & $0 \%$ & \\
\hline $\mathrm{E}$ & $60 \%$ & $76 \%$ & $38.2 \%$ & \\
\hline $\begin{array}{l}\text { Patent ductus arteriosus, minimal diameter, } \\
\text { median (range) }[\mathrm{mm}]\end{array}$ & $1.45(0.5-3.7)$ & $1.1(0.5-3.6)$ & $2.1(1.3-5.5)$ & $<0.001^{\star}$ \\
\hline $\begin{array}{l}\text { Patent ductus arteriosus, maximal diameter, } \\
\text { median (range) }[\mathrm{mm}]\end{array}$ & $4.75(1.5-11.4)$ & $3.1(0.5-7.2)$ & $4.3(2.1-9.6)$ & $<0.001^{\star}$ \\
\hline $\begin{array}{l}\text { Patent ductus arteriosus, length, } \\
\text { median (range) }[\mathrm{mm}]\end{array}$ & $7.6(3.2-20)$ & $7.3(2.8-14)$ & $8.3(2-13.5)$ & $0.58^{\star}$ \\
\hline
\end{tabular}


Adverse events in the ADO-1 group included transient second-degree heart block during catheter manipulation and pulse loss in another patient treated with heparin and thrombolytics with successful recanalization.

The distribution of PDA type based on the Krichenko classification and the PDA measurements are also shown in Table I. Type E was the most common type in this cohort (56.2\%) and was most frequent type in the Coils group. As expected, the PDA minimal diameter and maximal diameters were significantly smaller in the Coils group than in the Nit-Occlud and ADO-1 groups. The median PDA minimal diameter in the Nit-Occlud group was $1.45 \mathrm{~mm}(0.5-$ $3.7 \mathrm{~mm}$ ) compared to $1.1 \mathrm{~mm}(0.5-3.6)$ in the Coils group and $2.1(1.3-5.5 \mathrm{~mm})$ in the ADO-1 group. The PDA length was, however, similar among the three groups.

The incidence of the immediate angiographic residual shunt was significantly higher in the Nit-Occlud group at $70 \%$, compared to $26 \%$ and $59 \%$ in the Coils and ADO-1 groups, respectively (Table II). All angiographic residual shunts were tiny or small. The echocardiographic residual shunt was seen in only $10 \%$ of Nit-Occlud cases at discharge $4 \mathrm{~h}$ later. This incidence is similar to the residual shunts seen at discharge echo in the other two groups.
In other words, complete closure was documented in $90 \%$ of the Nit-Occlud group, $87 \%$ of the Coils group and $91 \%$ of the ADO-1 group on the day of the procedure. At the 2-month follow-up, complete closure rates were $100 \%$ in the Nit-Occlud group, $88 \%$ in the Coils group and $94 \%$ in the ADO-1 group. At 6 months, complete closure was noted in $97 \%$ of the AD0-1 group. In the Coils group, however, there were 3 subjects who still had a persistent shunt at 6 months, 2 of whom needed a second device to achieve complete closure.

Further review of the Nit-Occlud cohort was performed to separate out the characteristics between those who had an angiographic residual shunt vs. those who did not (Table III). The subjects with the residual shunt in the Nit-Occlud cohort had larger PDA than those without a residual shunt. The Nit-Occlud device can position itself in the ductus in different orientations based on the length of the ductus and its diameter (Figures 1 and 2). The device ductal angle ranged from zero to 88.1 degrees (Table III, Figure 2). The device orientation, device-ductal angle, and the number of loops in the pulmonary artery did not correlate with the degree of immediate angiographic residual shunt on angiogram (Table III).

Table II. Residual shunt after PDA device closure among the three groups

\begin{tabular}{|c|c|c|c|c|}
\hline Parameter & $\begin{array}{l}\text { Nit-Occlud } \\
\quad(n=30)\end{array}$ & $\begin{array}{c}\text { Coils } \\
(n=25)\end{array}$ & $\begin{array}{l}\text { ADO-1 } \\
(n=34)\end{array}$ & $P$-value \\
\hline \multicolumn{4}{|c|}{ Immediate angiographic residual shunt: } & 0.005 \\
\hline None & $30 \%$ & $74 \%$ & $41 \%$ & \\
\hline Tiny/Small & $70 \%$ & $26 \%$ & $59 \%$ & \\
\hline \multicolumn{4}{|c|}{ Residual shunt at discharge echo: } & 0.07 \\
\hline None & $90 \%$ & $61 \% *$ & $79.4 \% *$ & \\
\hline Tiny/Small & $10 \%$ & $8.7 \%$ & $8.8 \%$ & \\
\hline \multicolumn{4}{|l|}{ Residual shunt at follow-up: } & 0.15 \\
\hline At 2 months follow-up & $10 \%$ & $12 \%$ & $6 \%$ & \\
\hline At $\geq 6$ months follow-up & $0 \%$ & $12 \%$ & $3 \%$ & \\
\hline
\end{tabular}

Table III. Comparison of the subjects with and without an immediate angiographic residual shunt in Nit-Occlud cohort

\begin{tabular}{|c|c|c|c|}
\hline Parameter & $\begin{array}{c}\text { No angiographic residual } \\
\text { shunt }(n=9)\end{array}$ & $\begin{array}{l}\text { Angiographic residual } \\
\text { shunt }(n=21)\end{array}$ & $P$-value \\
\hline Patent ductus arteriosus minimal diameter, median (range) [mm] & $1(0.5-3.4)$ & $1.6(0.5-3.7)$ & $0.06^{*}$ \\
\hline Patent ductus arteriosus maximal diameter, median (range) [mm] & $3.4(1.5-6)$ & $5.6(1.5-11.4)$ & $0.004^{*}$ \\
\hline Device-ductal angle, median (range) $\left[{ }^{\circ} \mathrm{C}\right]$ & $0(0-75)$ & $41.9(0-88.1)$ & $0.51^{\star}$ \\
\hline \multicolumn{4}{|l|}{ Number of loops in pulmonary artery: } \\
\hline$\leq 1$ & $55 \%$ & $62 \%$ & $0.53^{* *}$ \\
\hline$>1$ & $45 \%$ & $38 \%$ & \\
\hline
\end{tabular}

*Independent sample Kruskal-Wallis test, ${ }^{* *} \chi^{2}$ test. 


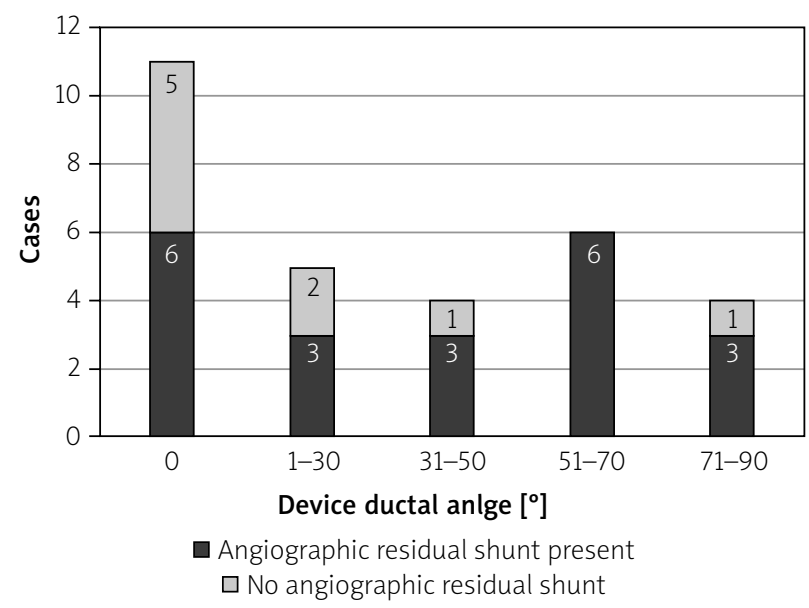

Figure 2. Distribution of device-ductal angle in the Nit-Occlud group. $\mathrm{X}$-axis shows the device-ductal angle in degrees and $Y$-axis represents the number of cases. The proportion of subjects with an angiographic residual shunt (black) and without an angiographic residual shunt (gray) are shown in the bar graphs

\section{Discussion}

Immediate angiographic residual shunts were seen commonly in our cohort of PDA closures using the Nit-Occlud device. The immediate angiographic shunt rate of $70 \%$ observed in the Nit-Occlud cohort of our study is similar to the $46.5 \%$ to $87.5 \%$ previously reported with the Nit-Occlud PDA device [8,9]. Turner et al. [11] and Lashus et al. [12] reported that an immediate residual angiographic shunt with traditional Gianturco or Flipper coils was associated with a residual shunt at follow-up. For that reason, there may be concerns that a similar residual angiographic shunt with the Nit-Occlud device may persist, requiring further treatment. Reassuringly, we found a residual shunt of $10 \%$ on the predischarge echocardiogram and a $90 \%$ rate of echocardiographic closure over $4 \mathrm{~h}$ after implantation. Our study is the only report comparing immediate angiographic shunt to echocardiographic shunt within $4 \mathrm{~h}$ with the Nit-Occlud PDA device. At the 2-month follow-up, the 3 remaining residual shunts had closed. This closure rate persisted in all patients who had returned for 6 months of follow-up. The results from the combined U.S. multicenter pivotal and continued access trial using the Nit-Occlud PDA device suggested that complete echocardiographic closure at 6 months persisted at the 12-month follow-up, with a composite echocardiographic closure rate of $96.8 \%$ at 12 months [4].

The sizes of the PDAs in the Nit-Occlud cohort were larger than those in the Coils group and similar to those closed with ADO-1. The largest PDA that can be closed with this Nit-Occlud is a PDA with a minimal diameter of $4 \mathrm{~mm}$ or less; PDAs larger than that were closed with an ADO-1 device in this study. Despite the size differences, the technical success rates were high in all 3 groups. There was a significant association between a larger PDA diameter and the presence of an immediate angiographic residual shunt, suggesting that only the smallest ductus left the catheterization laboratory without an angiographic residual shunt (Table III).

The mechanism of an angiographic residual shunt in the majority of the Nit-Occlud group was due to a small residual resembling a 'Jet' either through the center or adjacent to the device, depending on the orientation. This is in contrast to the residual shunt described with the ADO-1 device, where it is usually a result of 'foaming' through the device [13]. The angiographic appearance of the residual shunt in those that persisted at discharge and those that did not was similar, pointing to the inability to predict predischarge echocardiographic residual shunt from angiographic appearance. The Nit-Occlud device has no fibers attached, unlike the Flipper and MReye Coils, and relies on the thrombotic properties of the Nitinol surface and the bulk of the coils. The ADO-1 device, on the other hand, has polyester fabric inside the nitinol mesh to aid thrombosis and early closure. The absence of fibers in the Nit-Occlud PDA device may contribute to the higher immediate angiographic residual shunt; nevertheless, the rates of complete closure are excellent at follow-up. Heparin was used in all patients in this study irrespective of the type of device used; we do not believe this to be the reason for the higher incidence of the angiographic residual shunt, as suggested by Ghasemi et al. in their study [6].

The Nit-Occlud PDA device is a spiral coil with a cone in a cone configuration that is designed to be delivered with majority of the windings delivered in the aortic side and often one loop positioned in the pulmonary side [4]. We believe that the position of the rewound portion of the Nit-Occlud PDA device at the narrowest portion of the PDA is important for occlusion. However, the "proper" orientation of the Nit-Occlud PDA device along the PDA axis is not necessary for occlusion. The orientation of this device is quite variable with respect to the PDA, depending on the length and shape of the ductus. Sometimes the device orients parallel to the ductus, whereas other times, it is more perpendicular to it (Figure 1). We found that only $36.7 \%$ of devices were parallel to the axis of the PDA in our short series. The other $63.3 \%$ of Nit-Occlud devices were at angles of $12.5-88^{\circ}$ to the long axis of the PDA, but this did not influence occlusion (Figure 2). Similarly, there can sometimes be more than one loop in the pulmonary artery end. This is especially true in the larger PDAs, where more coils of the Nit-Occlud device can inadvertently be pulled into the pulmonary artery. The tension that can safely be placed on an ADO-1 device during implantation should be avoided with the Nit-Occlud PDA device to avoid pulling too much into the pulmonary artery. Once the aortic portion is in a good 
position, tension should be removed from the coils, and the pulmonary portion should be deployed by pulling the delivery catheter over the delivery system, even pushing the delivery system towards the aorta, to avoid pulling too much of the device into the pulmonary artery. The number of coils in the pulmonary artery can reduce the bulk of the rewound portion at the narrowed aortic ampulla. There was more than a single coil in the pulmonary artery in $41 \%$ of patients, but this did not affect the rates of angiographic or echocardiographic occlusion.

There are several limitations to this study. This is a retrospective review with a small sample size. The measurement of the device-ductal angle on a single lateral angiographic projection is not standard practice, but we believe it highlights the varying orientation of the device with respect to the ductus.

\section{Conclusions}

The rates of immediate, angiographic residual shunt are higher with the Nit-Occlud PDA device compared to the other Coils and the ADO-1 device. The orientation of the Nit-Occlud device with respect to the ductus does not impact the immediate angiographic residual shunt. Despite the higher immediate residual shunt rate, our study showed high closure rates within hours after device closure and a 100\% complete closure rate at 2 months. Angiographic residual shunt should not be a deterrent to using this device.

\section{Conflict of interest}

The authors declare no conflict of interest.

\section{References}

1. Fyler DC. Patent ductus arteriousus. In: Nadas' Pediatric Cardiology. Hanley \& Belfus, Philadelphia, PA 1992; 525-34.

2. Feltes TF, Bacha E, Beekman RH 3rd, et al. Indications for cardiac catheterization and intervention in pediatric cardiac disease: a scientific statement from the American Heart Association. Circulation 2011; 123: 2607-52.

3. Porstmann W, Wierny L, Warnke H. Der verschluss des ductus arteriosus persistens ohne thorakotomie: 1 , mitteilung [The closure of the patent ductus arteriosus without thoracotomy (preliminary report)]. Thoraxchirurgie 1967; 15: 199-203.

4. Moore JW, Greene J, Palomares S, et al. Results of the combined U.S. multicenter pivotal study and the continuing access study of the Nit-Occlud® PDA Device for percutaneous closure of patent ductus arteriosus. J Am Coll Cardiol Intv 2014; 7: 1430-6.

5. U.S. FDA. Summary of safety and effectiveness data (SSED) for the Nit-Occlud® PDA. August 26, 2013. Available at: http://www. accessdata.fda.gov/cdrh_docs/pdf12/P120009b.

6. Ghasemi A, Pandya S, Reddy SV, et al. Trans-catheter closure of patent ductus arteriosus-What is the best device? Catheter Cardiovasc Interv 2010; 76: 687-95.

7. Krichenko A, Benson LN, Burrows P, et al. Angiographic classification of the isolated, persistently patent ductus arteriosus and implications for percutaneous catheter occlusion. Am J Cardiol 1989; 63: 877-80.
8. Celiker A, Aypar E, Karogöz T, et al. Transcatheter closure of patent ductus arteriosus with Nit-Occlud ${ }^{\circledR}$ coils. Catheter Cardiovasc Interv 2005; 65: 569-76.

9. Gamboa R, Mollón FP, Ríos-Méndez RE, et al. Patent ductus arteriosus closure using a new device: the Nit-Occlud ${ }^{\circledR}$ device. Rev Esp Cardiol 2007; 60: 445-8.

10. Lloyd TR, Fedderly R, Mendelsohn AM, et al. Transcatheter occlusion of patent ductus arteriosus with Gianturco coils. Circulation 1993; 88: 1412-20.

11. Turner DR, Forbes TJ, Epstein ML, Vincent JA. Early reopening and recanalization after successful coil occlusion of the patent ductus arteriosus. Am Heart J 2002; 143: 889-93.

12. Lashus AG, Prieto LR, Latson LA. Are ductus arteriosus reopening and late closure following transcatheter coil placement significant issues. J Am Coll Cardiol 1999; 33: 527A.

13. Masura J, Walsh KP, Thanopoulous B, et al. Catheter closure of moderate- to large sized patent ductus arteriosus using the new Amplatzer duct occluder: immediate and short term results. J Am Coll Cardiol 1998; 31: 878-82. 